\title{
Studying Interstellar Shells in our Milky Way Galaxy ${ }^{1}$
}

\author{
Dr. Shauna Sallmen \\ University of Wisconsin - La Crosse
}

\begin{abstract}
The effects of generations of stars within the interstellar medium (ISM) have produced a turbulent, multiphase medium whose evolution has shaped how solar systems like our own form within a galaxy. It is clear that the distribution of energy and material in the ISM of our Milky Way Galaxy is largely driven by the stellar winds and supernova explosions of dying stars, forming hot, expanding bubbles that sweep up shells of neutral material. These Interstellar shells gradually cool, slow down, and mix with the ambient ISM, however we still lack a complete picture of the relevant interstellar processes. Studies of numerous shells are required to improve our understanding of their role in the evolution of our Galaxy. The author and undergraduate students have searched the SETHI radio survey database for new shells. In this project, the properties of 76 such shells were explored to evaluate their suitability for (1) follow-up multiwavelength imaging and (2) follow-up absorption-line spectroscopic studies. These investigations of interstellar shells will improve our understanding of their properties, characteristics, and interactions with the surrounding ISM.
\end{abstract}

\section{Introduction}

The space between the stars of our galaxy is filled with the low-density gas and dust of the interstellar medium (ISM). The ISM in the disk of our galaxy consists of several physical phases that range from dense and cold molecular gas to hot and fully ionized regions. This non-uniform gas exhibits a complex set of interacting structures. Shells, bubble-like features, "chimneys", and worms are driven by stellar winds and supernova (SN) explosions. The hot, ionized material ejected into the ambient ISM through these processes has been enriched in heavy elements through nuclear fusion inside of stars and during the supernova explosions. As the gas expands outward, it sweeps up cold neutral material into a shell surrounding a bubble of hot gas. Over time, the shells gradually expand and cool. The enriched gas mixes with the turbulent ambient ISM, portions of which eventually cool into dense molecular clouds that are the birthplace of new solar systems. Understanding these processes has a direct impact on our studies of (1) galaxy evolution during the history of the universe, and (2) the history of our own solar system. This topic therefore links explicitly to the objectives of the NASA Science Mission Directorate (SMD), which seeks to scientifically understand the solar system ${ }^{2}$, "[...] the universe and to understand the nature of the strange phenomena that shape it."

\footnotetext{
${ }^{1}$ Financial support for this research was provided by the Wisconsin Space Grant Consortium, the Research Corporation, and the National Science Foundation.

${ }^{2}$ http://nasascience.nasa.gov/about-us
} 
Thus, the physical state and dynamics of the ISM in galaxies determine how energy and matter released by stars are redistributed throughout the Universe, and incorporated into new solar systems. It is therefore critical to first understand the interstellar gas in the Milky Way, which can be observed with greater sensitivity and resolution than other galaxies. Although the physical state and evolution of gas phases in our galaxy have been broadly explained, a detailed understanding has not been achieved. In the three-phase model of McKee \& Ostriker (1977), random supernovae result in a turbulent ISM in which hot, low-density gas fills $\sim 70 \%$ of the volume, surrounding warm and cold clouds. In the galactic fountain model of Shapiro and Field (1976), hot gas rises out of the galactic plane, cools, then falls back into the galactic plane. Superbubbles (caused by clusters of supernovae) can break out of the galactic plane, enhancing the possibility of a galactic fountain. Cox \& Slavin (1992) incorporated the effects of magnetic fields in their models of supernovae evolution. The results indicate that the filling factor of hot gas is substantially less than original estimates. However, the Slavin \& Cox (1993) picture assumes that the disrupting influence of supernova is small, a view which is controversial (see Cox 2005). There are still many outstanding problems with these (and all other current) models of the ISM (See Cox (2005) and Ferrière (2001) for detailed reviews of these issues).

Intensive study of the interactions of particular supernova remnants (SNRs) with their environment will improve our understanding of the overall processes in the ISM. According to various models, shells of material from old supernova remnants should be ubiquitous (Slavin \& Cox 1993, Shelton 1996). A detailed knowledge of their physical properties is therefore essential to understanding the important processes of the ISM. Nevertheless, the energy inputs of shells are imperfectly understood, since "there is no one-to-one relationship of shells with young star clusters and associations" (Heiles 1984). Most well-studied shells are either quite close to the Sun, or are extremely large and distant. The number and size of large shells in the outer galaxy cannot yet be explained by the expected level of star formation in those regions, despite consideration of numerous alternatives (see McClure-Griffiths et al 2002). To make progress, we must increase the number of well-studied galactic shells.

Clearly, a first step is to identify for follow-up study neutral hydrogen (HI) shells which have previously been neglected. Recent radio surveys of neutral hydrogen with unprecedented sensitivity and angular resolution such as the Search for Extraterrestrial HI (SETHI; Korpela et al 2002), and the Southern Galactic Plane Survey (SGPS; McClure-Griffiths et al 2005) have resulted in the discovery of new shells of neutral gas (McClure-Griffiths et al 2001, Korpela et al 2004). Newer surveys such as the Parkes Galactic All-Sky Survey (GASS; McClure-Griffiths et al 2009) and the Galactic Arecibo L-Band Feed Array survey (GALFA-HI; Stanimirovic et al. 2006, Peek \& Heiles 2008) provide data at even greater sensitivity and angular resolution. These rich new resources are resulting in the identification of previously neglected interstellar shells ripe for further study (e.g. Sallmen et al. in preparation)

For best results, each shell must be investigated using a variety of methods. Because "the multiwavelength whole is far more than the sum of its parts" (Heiles, Haffner \& Reynolds 1999), the interaction of SNRs with the surrounding ambient ISM must be studied at multiple wavelengths. When combined with $21-\mathrm{cm}$ radio images of the cold, neutral gas in a shell, H- $\alpha$ images showing the warm, ionized hydrogen gas and X-ray images displaying the structure of hot gas will give us a more complete picture of how that shell is evolving and mixing with the ambient medium. 
In addition, absorption lines in the spectra of stars lying beyond the shell can tell us about the location, physical conditions and expansion (because of the Doppler effect) of the shell. Such absorption-line studies provide more accurate distance determinations for the shells, as well as a crucial diagnostic of the physical conditions of the gas within the shell. HI distance estimates derived by interpreting $\mathrm{HI}$ emission with a standard galactic rotation model are necessarily uncertain. By comparing absorption spectroscopy of stellar continuum sources with increasing sightline distances, the physical location and distance of absorbing gas at various velocities can be confidently identified. This allows their association with actual interstellar structures. For example, this technique was used to confirm the distance and origin of the IC 443 SNR (Welsh \& Sallmen 2003). More accurate distance estimates to HI shells also directly affect their derived sizes, and thus improve determinations of the energy required to produce such large interstellar features. In addition, the velocities of approaching and receding absorbing material can be used to determine the kinematic properties of the expanding shell.

Finally, by comparing absorption caused by both neutral species (reflecting cold gas) and ionized species (reflecting warmer gas), we can probe the physical conditions of gas within the shell. For example, the ionization state and column density of gas in the Shajn 147 SNR were determined using absorption-line studies (Sallmen \& Welsh 2004). The NaI doublet absorption lines (5890 $\& 5896 \AA$ ) are ideal for this work because they probe neutral gas associated with cold HI. The CaII lines (3933 \& 3968 $\AA$ ) trace slightly warmer, ionized gas. These absorption lines reveal both the velocity structure and column density of the low-ionization interstellar gas lying foreground to an emitting continuum object. The $\mathrm{N}(\mathrm{NaI}) / \mathrm{N}(\mathrm{CaII})$ ratio is also an important physical diagnostic of the gas. When it is small (e.g. $<0.5$ ), this is interpreted as being indicative of the presence of warm $(7000 \mathrm{~K})$ interstellar gas in which Ca dust grains have been destroyed by shocks or strong ionization effects (e.g. Bertin et al 1993). It is true that absorption measurements from stars will only probe a few pencil beams within each shell, so variable conditions within the shells will cause spatial variations in our results. Comparison of these absorption measurements with existing emission measurements at a variety of wavelengths will alleviate this issue by allowing us to assess the conditions for each sightline.

One example of a well-studied nearby SNR is the Cygnus Loop, at a distance of $\sim 440 \mathrm{pc}$. Detailed investigation of the velocity structure of the neutral hydrogen (Leahy 2003), optical absorption-line studies of the cool, neutral gas (Welsh, Sallmen, Sfeir \& Lallement 2002), investigations of the shock using X-ray spectroscopy (Levenson, Graham \& Walters 2002), OVI emission (Raymond et al 2003), HST imaging (Levenson \& Graham 2001) and H- $\alpha$ imaging (Fesen, Kwitter \& Downes 1992) have all contributed to our understanding of this object. Similar exhaustive multi-wavelength studies of a wide variety of SNRs at varying galactic distances are necessary for a complete understanding of the processes in the galactic interstellar medium.

\section{Methods}

Over the past several years, the author has involved undergraduate students in searching for interstellar shells, and studying their properties through images at a variety of wavelengths. With the help of undergraduate students, the SETHI neutral hydrogen dataset has been searched, producing a list of shell-like features which are missing from previous catalogs (Sallmen et al., in preparation). 
The goal of this project is to select newly identified shells which are suitable for further study:

- Explore existing archival images at multiple wavelengths for shells of potential interest for follow-up multi-wavelength observations

- Use kinematic distance information to identify shells suitable for absorption-line work

- Select appropriate stellar targets for each shell, and explore availability of absorption-line data for these stars

With the help of undergraduate students, archival images at multiple wavelengths were explored for 76 shells that were already identified using the SETHI radio data. Each shell's coordinates, velocity and angular size were measured and checked against existing catalogs. For each shell not previously known, archival data was downloaded from the SkyView Virtual Observatory ${ }^{3}$. Images of the shell at were compared for $1 / 4 \mathrm{keV}$ X-rays (RASSband 1 \& RASSband 2 ), $\mathrm{H}-\alpha$ (HAlpha Composite Survey), $100 \mu \mathrm{m}$ infrared light (IRIS), as well as the $21-\mathrm{cm}$ neutral hydrogen from the SETHI data. These reveal the presence of hot $\left(\sim 10^{6} \mathrm{~K}\right)$ gas, warm ionized $\left(\sim 10^{4} \mathrm{~K}\right)$ hydrogen gas, warm interstellar dust, and cold $(\sim 100 \mathrm{~K})$ neutral hydrogen gas, respectively. Shells showing clear shell-like features and multiple wavelengths, and/or correlations or anticorrelations between images are more suitable for further multi-wavelength analysis, as they give a more complete picture of the shell's evolution and mixing into the ISM. Each shell was assigned a ranking 1-3 describing its potential for multi-wavelength follow-up. For details of the procedure, see the proceedings paper by E. Tennyson (this volume).

To get the most out of such multi-wavelength studies, the distance to the shell must be known. At some galactic longitudes, the HI emission velocity can be interpreted in light of a galactic rotation model to estimate the distance. For each shell in our list, the radial velocity \& range of velocities were compared with the rotation curve of Brand \& Blitz (1993) to estimate its distance. This kinematic distance was then used to estimate the shell's physical size. Based on existing catalogs, HI shells are typically 100 to 300 pc in size (Ehlerová \& Palouš 2005). We therefore wish to focus our attention on those shells with relatively well-defined kinematic distances which yield shell size estimates less than 300pc in size. These preliminary distance estimates also allow us to identify more appropriate potential stellar targets for absorption-line studies of these shells.

Several considerations apply when determining shells of interest for absorption-line spectroscopic studies. Such studies are particularly applicable to nearby shells with large expansion velocities. Nearby shells are more likely to have sufficient foreground and background target stars. Shells which have large expansion velocities (based on HI emission from the approaching / receding walls) must be selected to ensure that the absorption components due to the shell are well-separated in the spectrum from those due to the ambient ISM.

The existence of appropriate stellar targets is also an important consideration. Hot, luminous (early-type $\mathrm{O}$ and B) stars are the best background continuum sources for $\mathrm{NaI}$ and $\mathrm{CaII}$ spectroscopic studies, and must be present at several distances along the line of sight towards the shell. The preliminary kinematic distance estimate allows us to identify the most appropriate potential stellar targets for each shell. For a shell expansion velocity $\geq 20 \mathrm{~km} / \mathrm{s}(\Delta \mathrm{v} \geq 40 \mathrm{~km} / \mathrm{s})$, we require spectral resolution $\mathrm{R} \sim 5 \mathrm{~km} / \mathrm{s}$ in order to separate absorption components from the

\footnotetext{
${ }^{3}$ http://skyview.gsfc.nasa.gov
} 
main (and saturated) central line-of-sight absorption. Suitable spectra are typically taken with instruments such as the Hamilton echelle on the University of California Lick CAT, or the Giraffe echelle spectrograph mounted on the 1.9m Radcliffe telescope of the South African Astronomical Observatory. High-velocity components are easily detectable in $7^{\text {th }}$ magnitude stars out to $\sim 0.5 \mathrm{kpc}$ using the Lick CAT. For more distant stars, background early-type target stars $(\mathrm{Mv}=6.0-9.5)$ with distances $0.5-3 \mathrm{kpc}$ are observable on a $2-\mathrm{m}$ class telescope. On such a telescope it is possible to record the spectrum of an OB star of $\mathrm{Mv} \sim 9.0$ at a resolving power of 60,000 in one hour with a $\mathrm{S} / \mathrm{N}$ ratio $>40: 1$. Suitable early-type stellar targets can be extracted from online stellar catalogs ${ }^{4}$ for each selected shells. Shells with few available stellar targets are eliminated from further consideration for follow-up absorption-line work.

\section{Results}

Of the 76 shells examined, 21 were determined to be found in the existing catalog of Ehlerová \& Palouš (2005). Based on the existing multi-wavelength data, 23 of the remaining 55 shells are unsuited to follow-up multi-wavelength studies, 23 may be suited to follow-up multi-wavelength studies, and 9 are likely targets for such follow-up studies.

Table 1 shows the properties of these 9 shells, based on the SETHI data. Column 1 gives the name of the shell, based on its Galactic coordinates and velocity at which it is most "shell-like". Columns 2 and 3 give the equatorial coordinates of the shell, while Column 4 gives the velocity range over which the shell is visible, and Column 5 gives the mean angular diameter of the shell. The final column gives the size estimate based on the mean angular diameter and kinematic distance estimates. Kinematic distance information may be found in Table 3 of Tennyson (proceedings this volume).

Table 1: Shells most suited to multi-wavelength follow-up observations

\begin{tabular}{|c|c|c|c|c|c|}
\hline $\begin{array}{c}\text { Shell ID } \\
\text { GSH lll+bb+vvv }\end{array}$ & $\begin{array}{c}\text { RA } \\
\text { hh } \mathrm{mm}\end{array}$ & $\begin{array}{c}\text { Dec } \\
\mathbf{o}\end{array}$ & $\begin{array}{c}\boldsymbol{\Delta} \mathbf{k} \\
\mathrm{km} / \mathrm{s}\end{array}$ & $\begin{array}{c}\text { Mean Ang. } \\
\left.\text { Diam. } \mathbf{(}^{\mathbf{}}\right)\end{array}$ & $\begin{array}{c}\text { Size } \\
\text { pc }\end{array}$ \\
\hline GSH029+38+005 & 1628 & 13.75 & 4.64 & 3.1 & $29_{-24}^{+23}$ or $990_{-30}^{+20}$ \\
\hline GSH034+20+011 & 1742 & 9.5 & 10.83 & 2.8 & $41_{-27}^{+25}$ or $690_{-20}^{+30}$ \\
\hline GSH044+38+022 & 1645 & 24.75 & 6.21 & 1.9 & $<16$ or $51_{-2}^{+2}$ \\
\hline GSH114-54-005 & 0030 & 8.5 & 9.28 & N/A & N/A (incomplete) \\
\hline GSH208+32+006 & 0843 & 18 & 15.46 & 5.8 & $110_{-110}^{+150}$ \\
\hline GSH213+28+012 & 0834 & 12 & 12.38 & 3.7 & $110_{-60}^{+70}$ \\
\hline GSH274+74-006 & 1219 & 12.5 & 10.82 & 6.4 & $<890$ \\
\hline GSH294+76+000 & 1243 & 13.25 & 9.28 & 4.1 & $<950$ or $>1130$ \\
\hline GSH325+78-001 & 1310 & 15.75 & 7.74 & 8.4 & $290_{-290}^{+1130}$ \\
\hline
\end{tabular}

Based on the kinematic distance and resulting size estimates for these 9 shells, there are 4 shells with relatively well-defined distance / size estimates consistent with the expected 100-300pc maximum shell size. These entries are bold-faced in Table 1, and represent the 4 shells most suited to follow-up multi-wavelength observations.

\footnotetext{
${ }^{4}$ Accessed via the SIMBAD astronomical database (http://simbad.u-strasbg.fr/simbad/ )
} 
For each shell in Table 1, online stellar catalogs were used to search for O \& B stars in a rectangular region containing the shell. The results are shown in Table 2 . The left-hand table shows the results for the shells deemed most interesting based on both archival multi-wavelength image exploration \& kinematic distance estimates. None of these have enough potential stellar candidates to merit further consideration for absorption-line studies. The right-hand table shows the results for the remaining shells highlighted through the exploration of archival multiwavelength images. Although these have less well-defined kinematic distance estimates, two of these do have sufficient stellar target candidates to merit further consideration for absorption-line studies.

Table 2: Candidate Stellar Targets

\begin{tabular}{|c|c|}
\hline Shell ID & \# O \& B stars \\
\hline GSH029+38+005 & 3 \\
\hline GSH034+20+011 & 2 \\
\hline GSH044+38+022 & 1 \\
\hline GSH213+28+012 & 3 \\
\hline
\end{tabular}

\begin{tabular}{|c|c|}
\hline Shell ID & \# O \& B stars \\
\hline GSH114-54-005 & 8 \\
\hline GSH208+32+006 & 8 \\
\hline GSH274+74-006 & 4 \\
\hline GSH294+76+000 & 0 \\
\hline GSH325+78-001 & 2 \\
\hline
\end{tabular}

\section{Future Work}

Multi-wavelength image exploration based on archival data must still be completed for at least 7 more newly identified shells, to complete the selection of shells suitable for multi-wavelength follow-up studies.

Stellar target candidate identification must be completed for all of the newly identified shells, focusing on the 23 which are potentially interesting based on the comparison of archival images at multiple wavelengths. In an ideal world, we would perform absorption-line studies on shells which are also suited to multi-wavelength imaging studies. However, lack of availability of stellar targets may force us to do absorption-line studies of some shells which are less suited to multi-wavelength imaging studies.

Once suitable targets are selected, existing and newly obtained absorption-line spectra will be used to analyze the physical properties of the interstellar shells, and to refine their distances.

Absorption-line spectra have likely already been acquired for some of these stellar targets. For example Welsh et al. (2010) contains $\mathrm{NaI}$ and CaII measurements to hundreds of bright, relatively nearby stars, and colleagues active in this area will be contacted to gain access to the relevant spectra. Absorption-line spectra of stellar continuum targets are used to analyze the properties of gas in an intervening interstellar shell. For some targets, the gas cloud velocity, cloud dispersion parameter, and column density may already be available in the literature. Other existing absorption-line spectra will be searched for interstellar absorption features formed in the expanding nebular shell gas. The observations will be of sufficient spectral resolving power to separate absorption components due to shell expansion from the central line-profiles (formed from line-of-sight gas in the ISM between the Sun and a shell, and usually saturated). Overall, improved shell distance estimates will help constrain the energy requirements and spatial distributions of these objects. This will lead to an improved understanding of their formation mechanism and interaction with each other and the ambient ISM in our galaxy. 


\section{Acknowledgements}

I acknowledge the support of the Wisconsin Space Grant Consortium in carrying out this research, and the Research Corporation and National Science Foundation for supporting my research program.

I acknowledge the use of NASA's SkyView facility (http://skyview.gsfc.nasa.gov) located at NASA Goddard Space Flight Center, and the SETHI database (http://setiathome.berkeley.edu/ korpela/sethi), which uses data acquired at Arecibo Observatory. The Arecibo Observatory is part of the National Astronomy and Ionosphere Center which is operated by Cornell University under a Cooperative Agreement with the National Science Foundation.

I would like to acknowledge the participation in this project of undergraduate students Elizabeth Tennyson and Cheuk Man Lo, as well as the past contributions of Brooke Baldwin and Kurt Grunwald, who searched for shells in the SETHI data. 


\section{References}

Bertin, P., Lallement, R., Ferlet, R., \& Vidal-Madjar, A. 1993, A\&A, 278, 549

Brand, J. \& Blitz, L. 1993, A\&A, 275, 67

Cox, D.P. \& Slavin, J. 1992, ApJ, 392, 131

Cox, D.P. 2005, Ann. Rev. Astron. Astrophys., 43, 337

Ehlerová, S. \& Palouš, 2005, A\&A, 437, 101

Ferrière, K. 2001, Rev. Mod. Phys., 73, 1031

Fesen, R., Kwitter, K. \& Downes, R..1992, AJ, 104, 719

Heiles, C. 1984, ApJS, 55, 585

Heiles, C., Haffner, L.M. \& Reynolds, R.J. 1999, in ASP Conf. Series 168, p211, Ed. A.R. Taylor, T.L.Landecker \& G. Joncas (San Francisco: ASP)

Korpela, E. J., Demorest, P., Heien, E., Heiles, C. \& Werthimer, D. 2002, “SETHI@Berkeley - A Piggyback 21-cm Sky Survey at Arecibo" in ASP Conf. Series 276, p100, Ed. A.R. Taylor, T.L. Landecker \& A. Willis (San Francisco: ASP)

Korpela, E. J., Demorest, P., Heien, E., Heiles, C., \& Werthimer, D. 2004, in Proceedings from the 2003 June Galactic Tertulia How Does the Galaxy Work, p 97, Ed. E.J. Alfaro, E. Perez \& J. Franco (Dordrecht: Kluwer Academic Publishers)

Leahy, D.A. 2003, "Structure of the HI near the NE Limb of the Cygnus Loop", ApJL, 586, 224

Levenson, N. \& Graham, J. 2001, ApJL, 559, 948

Levenson, N., Graham, J. \& Walters, J. 2002, ApJ, 576, L798

McClure-Griffiths, N. M., Green, A.J., Dickey, J.M., et al. 2001, ApJ, 551, 394

McClure-Griffiths, N. M., Dickey, J. M., Gaensler, B. M., \& Green, A. J. 2002, ApJ, 578, 176

McClure-Griffiths, N. M., Dickey, J. M., Gaensler, B. M., et al. 2005, ApJS, 158, 178.

McClure-Griffiths, N. M., Pisano, D. J., Calabretta, M. R., et al. 2009, ApJS, 181, 398

McKee, C. \& Ostriker, J. 1977, ApJ, 218, 148

Peek, J.E.G., \& Heiles, C. 2008 arXiv:0810.1283

Raymond, J.C., Ghavamian, P., Sankrit, R., Blair, W. P. \& Curiel, S. 2003, ApJ, 584, 770

Sallmen, S. and Welsh, B.Y. 2004, A\&A, 426, 555

Shapiro, P. \& Field, G., 1976, ApJ, 205, 762

Slavin, J. \& Cox, D.P. 1993, ApJ, 417, 187

Shelton, R.L. 1996, “The ISM in Our Galaxy”, PhD Thesis, Univ. of Wisconsin-Madison

Stanimirovic, S., Putman, M., Heileis, C., et al. 2006, ApJ, 653, 1210

Welsh, B.Y., Sallmen, S., Sfeir, D. \& Lallement, R. 2002, A\&A, 391, 705

Welsh, B.Y. \& Sallmen, S., 2003, A\&A, 408, 545

Welsh, B.Y., Lallement, R.,Vergely, J.-L., \& Raimond, S. 2010, A\&A, 510, A54 